American Journal of Infectious Diseases 3 (1): 27-35, 2007

ISSN 1553-6203

(C) 2007 Science Publications

\title{
The Value of Serologic Tests for Diagnosis and Follow up of Patients having Brucellosis
}

\author{
${ }^{1}$ Nidia E. Lucero, ${ }^{1}$ Sandra M. Ayala, ${ }^{1}$ Gabriela I. Escobar and ${ }^{2}$ Néstor R. Jacob \\ ${ }^{1}$ National Laboratories and Institutes of Health Administration Dr. C.G.Malbrán (ANLIS) \\ Avda. Velez Sarsfield 563, 1281 BUENOS AIRES, Argentina \\ ${ }^{2}$ Infectology Department, Austral University Hospital, BUENOS AIRES, Argentina
}

\begin{abstract}
Though diagnosis of human brucellosis is accurate when the causal agent is isolated, this procedure is not always successful and the most of patients are diagnosed on the basis of rising titres of antibodies in serum. The classical tests used for detection of antibodies to S-Brucella sp., include Rose Bengal (RBT), buffered plate antigen (BPAT), serum agglutination (SAT), 2-mercapto-ethanol (2MET) and complement fixation (CFT). The modern methods are based on primary binding assays of which a competitive enzyme immunoassay (CELISA) and fluorescence polarization (FPA) are the best developed. For antibodies to R-Brucella sp. a rapid slide agglutination (RSAT) as screening and an indirect ELISA (IELISA) as confirmatory tests have been reported. We have selected 23 cases of human brucellosis that were followed up over a long period, to assess which test was most effective in detecting different stages of the disease. The patients were divided into five groups: "chronic" cases; relapses; infection acquired in a laboratory; patients presumptively infected with $B$. canis and cases with a long history of brucellosis. The results suggest that BPAT is a practical test that reduces non specific reactions and is more sensitive than RBT. SAT detects the acute form but cross reacts with other antibodies and the diagnostic end-point titre has not been satisfactorily established; 2MET should be discontinued because of its toxicity and the scant information it can add; CFT fails to detect the acute form and is technically complicated. CELISA correlate well with the clinical course and is useful to detect acute as well as "chronic" cases and FPA do not work in serum with high lipid content. RSAT and IELISA are useful tests for brucellosis caused by B. canis. A unique protocol for serologic diagnosis that uses robust tests would be of value to the surveillance and control the disease.
\end{abstract}

Key words: Brucellosis, human brucellosis, Brucella serology

\section{INTRODUCTION}

Although the most specific test for diagnosis of human brucellosis is the isolation of Brucella sp., its efficacy is low and therefore a negative blood culture cannot rule out the disease, particularly in "chronic" forms where negative cultures are frequent ${ }^{[1]}$. The complex immune response induced by Brucella involves both humoral and cellular effectors and provides valuable information about the stage and evolution of the disease. The antibodies appear early in the course of infection and may persist for months or even years, so that most patients are diagnosed on the basis of high or rising titres of specific antibodies in serum $^{[2]}$.

B. abortus, B. melitensis, B. suis and B. canis are pathogenic for humans, but while the first three species are smooth and contain O-polysaccharide on the cell surface the latter rough strain contain no measurable O- polysaccharide. Whole cell antigen, smooth lipopolysaccharide or cytoplasmic protein extraction are used as antigens for detection of antibodies to the three main species while rough lipopolysaccharide or protein are used as antigens for $B$. canis $^{[3]}$.

The classical serological tests used in human brucellosis for detection of antibodies to smooth Brucella strains, include Rose Bengal (RBT), buffered plate antigen (BPAT), serum agglutination (SAT), 2mercapto-ethanol (2MET) and complement fixation (CFT). The modern methods are based on primary binding assays of which ELISAs and fluorescence polarization (FPA) are the best developed. A competitive enzyme immunoassay (CELISA) has been shown to be a suitable test for human brucellosis ${ }^{[4]}$. This test uses a monoclonal antibody specific for a common and repeating epitope on the polysaccharide portion of the smooth lipopolysaccharide molecule of Brucella (S-LPS) to compete with antibody in the

Corresponding Author: $\quad$ Nidia E. Lucero, National Laboratories and Institutes of Health Administration Dr. C. G. Malbrán (ANLIS), Avda. Velez Sarsfield 563, 1281 BUENOS AIRES, Argentina 
sample. This results in a test with sensitivity (100\%) and specificity (99.7\%) higher than other assays and it eliminates cross-reactions with other antigens. Fluorescence polarization immunoassay (FPA) makes use of molecular rotational properties, measuring antibody binding to antigen directly, eliminating the need for separation procedures. The method has been applied to the detection of human antibodies resulting in a sensitive $(96.1 \%)$ and specific $(97.9 \%)$ test $^{[5]}$. For detection of antibodies to rough Brucella strains a rapid slide agglutination test (RSAT) and an indirect ELISA $(100 \% \text { sensitivity and specificity })^{[6]}$ that uses an antigen obtained from the (M-) variant of B. canis $^{[7]}$ have been developed as screening and confirmatory test respectively.

Because the serologic response varies with the stage of the disease, the tests used must help to diagnose the acute form and the relapsed and "chronic" cases, while also providing information about the evolution of the disease during and after antibiotic therapy. We now report the results of serologic and bacteriologic tests of 23 patients with different stages of brucellosis, most of which were followed up over a long period and discuss their contribution to patient management.

\section{MATERIALS AND METHODS}

Patients: The subjects were selected from about 10000 cases studied at the National Laboratories and Institutes of Health Administration "Dr.C.G.Malbrán" (ANLIS) from 1994 through 2006 after the implementation of the National Human Brucellosis Network (NHBN). The 23 patients selected were divided into five groups (4 groups of 5 cases and 1 group of 3 cases). The criteria of selection for this study were: a) in "chronic" cases, the duration of symptoms after therapy (between 20 and 60 months); b) relapses, confirmed by two or more isolations of Brucella sp.; c) for cases acquired in a laboratory, a positive blood culture at baseline; d) patients with $B$. canis isolation or in contact with dogs diagnosed with $B$. canis brucellosis and e) three cases selected because of a long history of brucellosis.

Therapy: The initial treatment approach was not standardized but the most cases received $1 \mathrm{~g}$ of IM streptomycin for 15 days and $100 \mathrm{mg}$ of oral doxycycline b.i.d per os for 45 days. Patients under 14 years old were treated with oral rifampin plus cotrimoxazole for six weeks. During the prolonged period of treatment patients received different schedules of antibiotics.
Serological methods: For detection of smooth Brucella antibodies: The classical BPAT, RBT, SAT, 2MET and CFT tests were run as described previously ${ }^{[8-10]}$ with antigens prepared at ANLIS using the B. abortus 11193 strain.

CELISA were run as per previous report ${ }^{[4]}$; the antigen (S-LPS from B.abortus 1119-3) and the MAb were standardized and supplied by the Brucellosis Centre of Expertise and OIE Reference Laboratory, Animal Diseases Research Institute (ADRI), Canada. The conjugate pre-adsorbed with bovine, equine and human serum protein was from Jackson Lab. The test is positive when $\% \mathrm{I}$ is equal to or above $28^{[4]}$.

Fluorescence polarization assay (FPA) was run with antigen supplied by the CNEA (National Atomic Energy Commission), Argentina, as described in a previous report ${ }^{[5]}$. The test is positive when $\mathrm{mP}$ is equal to or above $72^{[5]}$.

Control sera: As controls, positive and negative reference human sera were included in each classical test, in each CELISA plate and in each lot of 30 samples tested for FPA ${ }^{[4-5]}$.

For detection of rough Brucella antibodies: RSAT was the screening test and run as described previously ${ }^{[7,11]}$ with serial dilution in order to determine the final titre. The antigen was prepared at ANLIS with the strain (M-) variant of B. canis.

IELISA: The antigen was obtained from the (M-) variant of $B$. canis as described previously ${ }^{[7]}$ and the lyophilised protein $\mathrm{A} / \mathrm{G}$, horseradish peroxidase conjugated was from ImmunoPure, Pierce Lb. The final step added $100 \mathrm{ul}$ of chromogenic substrate $(4.0 \mathrm{mM}$

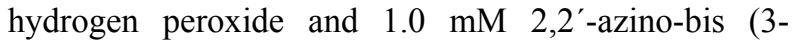
ethylbenz-thiazoline -6-sulfonic acid) diammonium salt in $0.05 \mathrm{M}$ citrate buffer, $\mathrm{pH} 4.5$ ). The plate was shaken continuously on an orbital shaker for $10 \mathrm{~min}$ and the $\mathrm{OD}_{414}$ then measured in a photometer (Labsystems Multiskan EX microplate reader) using $100 \mathrm{ul}$ chromogenic substrate in a plate as control for the microplate reader. The standard control serum used on each plate makes it possible to convert the optical density reading to percent positivity. The test is positive when $\% \mathrm{P}$ is equal to or above $27^{[6]}$.

Control sera: The strong, weak positive and negative control sera were include in each RSAT test and in each IELISA plate ${ }^{[6]}$.

Bacteriological studies: Brucella organisms were isolated by routine isolation techniques such as monophasic and biphasic blood culture, lysiscentrifugation and automated blood culture systems (BACTEC or BACT ALERT). For monophasic blood 
culture the commercial liquid medium Hemo Brucella (BRITANIA SA, Buenos Aires, Argentina) or a medium prepared in this laboratory containing NZ amine $1.5 \mathrm{~g}$, Primatone $0.5 \mathrm{~g}$, yeast extract $0.2 \mathrm{~g}$, dextrose $0.1 \mathrm{~g}$, sodium chloride $0.5 \mathrm{~g}$, sodium bisulphite $0.01 \mathrm{~g}$, sodium citrate $2.5 \mathrm{~g}$ and distilled water $100 \mathrm{ml}$ were used while for biphasic medium, Hemoline (bioMerieux, Marcy 1'Etoile, France) was mainly employed. Bone marrow, bone biopsy and epidural abscess were cultured by spreading material on solid medium and by inoculation into liquid medium. The samples were incubated at $37^{\circ} \mathrm{C}$ in $10 \% \mathrm{CO}_{2}$ and strains isolated were typed as recommended by the former ICBN Subcommittee on Taxonomy of the Genus Brucella $^{[9,12]}$ at ANLIS.

\section{RESULTS}

Laboratory acquired brucellosis: Table 1 shows five cases of laboratory-acquired brucellosis that we diagnosed. Case 1 was infected with $B$. melitensis biovar 1 after handling, on an open bench, a positive automated blood culture system from a patient admitted to the hospital. The symptoms: fever, weight loss and fatigue, disappeared after treatment and the serology was negative 10 and 15 months latter. Case 2 was diagnosed after isolation of the strain from blood by the Bact Alert system in a hospital laboratory after a consultation in which fever, asthenia and myalgias were the main symptoms. The strain isolated was sent to us for confirmation of genus and typing and a serum sample was requested at that time. The patient related have briefly visited a room where $B$. melitensis was manipulated. Neither signs nor symptoms of relapse were detected in the course of 12 months by the outpatient department. The serology titre of classical tests, CELISA and FPA decreased steadily. Case 3 occurred because of misidentification of the organism isolated by BACTEC from a boy admitted to the hospital that was manipulated on an open bench. The symptoms: fever, night sweats, leukopenia and anemia disappeared after treatment while the serology titre declined slowly. Cases 4 and 5 were engaged in identifying strains of clinical origin and were tested annually for brucellosis. Case 4 was investigated after inadvertently handling a Brucella strain isolated from a patient in whom disease was not suspected. The case that presented mild fever, fatigue and myalgias, was controlled clinically and serologically, presenting a favorable evolution with a slow decline in serologic titres. The first symptom of case 5 was persistent fever just after a cholecystectomy and B. suis biovar 1 was isolated from the blood culture. Despite two treatment schedules, 33 months since the disease was diagnosed, CELISA and FPA continue to present high values, while the patient had occasional episodes of mild fever.

Cases of "chronic" brucellosis: Table 2 shows five patients followed up for a long period. Case 6, a farmer, was admitted to the hospital with severe lumbalgia, spondylolisthesis Grade I, $4^{\text {th }} \mathrm{L}$ slip forward on $5^{\text {th }} \mathrm{L}$ and epidural abscess as main signs. Diagnosis was easily ascertained by isolation of the microorganism from blood, bone biopsy and epidural abscess as well as by serologic tests; on this basis, therapy was indicated. However, two and four months latter new abscesses appeared although the cultures were negative. Blood cultures were repeated 3 and 6 months after admission but were negative. The conventional serologic tests declined slowly after the first year but CELISA and FPA titres remained high 32 months latter. Case 7, a dairy farmer, with orquiepididymitis, arthritis of the knee and pancytopenia was admitted to the hospital and B. abortus biovar 1 was isolated from his blood. Although the patient was controlled clinically, bacteriologically and serologically throughout the antibiotic treatment, 22 months after admission another fever episode determined a second treatment scheme. Thirty four months later, CELISA and FPA titres continue to be high. Case 8 , a slaughterhouse worker, consulted because of an episode of fever, sweating and myalgias that occurred three months previously. The patient was symptom-free at the moment of the consultation, but was given antibiotic therapy for 45 days because of the serology titres. Nine months after this consultation he was asymptomatic but $B$. abortus biovar 1 was isolated from his blood and a new scheme of antibiotic therapy was indicated. At the end of this treatment the blood culture was negative but CELISA and FPA presented high titres. Case 9, another slaughterhouse worker, at the time he consulted, presented fatigue and arthralgias and stated that he had been diagnosed and treated for brucellosis one year before. Besides the serologic tests, three hemocultures were done and B. abortus biovar 1 was isolated. Because the symptoms continued and CELISA and FPA were very high, a second therapy scheme was prescribed. These titres were still high 24 months later, but the strain could not be recovered from blood cultures. Case 10, a slaughterhouse worker in direct contact with carcasses and viscera, was symptom-free at the moment of the first consultation. He was treated with antibiotics for 45 days, but 11 months later, CELISA and FPA indicated persistence of antibodies. 
Am. J. Infect. Dis., 3 (1): 27-35, 2007

Table 1: Serologic tests in five cases of laboratory acquired brucellosis

\begin{tabular}{|c|c|c|c|c|c|c|c|c|c|c|}
\hline Case & $\begin{array}{l}\text { Date } \\
\text { a }\end{array}$ & BPAT & RBT & SAT & 2MET & CFT & CELISA & FPA & $\begin{array}{l}\text { Source } \\
\text { isolation }\end{array}$ & Strain \\
\hline \multirow[t]{5}{*}{1} & 0 & Pos & Pos & 100 & Neg & 20 & 29 & 84 & Blood & B. melitensis bio 1 \\
\hline & 1 & Pos & Pos & 100 & $\mathrm{Neg}$ & 20 & 26 & E & & \\
\hline & 3 & Pos & Pos & 100 & $\mathrm{Neg}$ & 20 & 24 & 65 & Blood & Neg \\
\hline & 4 & Pos & Neg & 50 & Neg & 10 & 26 & 66 & & \\
\hline & 15 & Neg & Neg & Neg & Neg & Neg & 8 & 71 & & \\
\hline \multirow[t]{5}{*}{2} & 0 & & & & & & & & Blood & B. melitensis bio 1 \\
\hline & 1 & Pos & Pos & 1600 & 50 & 10 & 44 & $\mathrm{E}$ & & \\
\hline & 5 & Pos+/- & Neg & 50 & Neg & 5 & 5 & 52 & & \\
\hline & 11 & Neg & Neg & 25 & Neg & Neg & 6 & 53 & & \\
\hline & 12 & Neg & Neg & $25+/-$ & Neg & Neg & 8 & 59 & & \\
\hline \multirow[t]{4}{*}{3} & 0 & Pos & Pos & 6400 & 1600 & 640 & 69 & E & Blood & B.suis bio $1 \mathrm{a}$ \\
\hline & 2 & Pos & Pos & 400 & 50 & 20 & 41 & 69 & Blood & Neg \\
\hline & 4 & Pos & Neg & 50 & Neg & 10 & 28 & 45 & & \\
\hline & 27 & Neg & Neg & Neg & Neg & Neg & 25 & 60 & & \\
\hline \multirow[t]{7}{*}{4} & 0 & Neg & Neg & Neg & Neg & Neg & 0 & 45 & & \\
\hline & 54 & Pos & Pos & 400 & 50 & 80 & 58 & 73 & Blood & B.suis bio 1 \\
\hline & 56 & Pos & Pos & 400 & 50 & 80 & 51 & 79 & Blood & Neg \\
\hline & 57 & Pos & Pos & 200 & 25 & 40 & 45 & 75 & & \\
\hline & 67 & Pos & Pos+/- & 50 & Neg & 10 & 30 & 59 & & \\
\hline & 72 & Pos & Pos+/- & 50 & Neg & 10 & 32 & 65 & & \\
\hline & 81 & Pos & Pos+l- & 50 & Neg & 5 & 30 & 63 & & \\
\hline \multirow[t]{8}{*}{5} & 0 & Neg & Neg & Neg & Neg & Neg & 0 & 47 & & \\
\hline & 120 & Pos & Pos & 1600 & 200 & 320 & 69 & 70 & Blood & B.suis bio 1 \\
\hline & 122 & Pos & Pos & 200 & 100 & 40 & 41 & 79 & & \\
\hline & 123 & Pos & Pos & 400 & 100 & 40 & 56 & 78 & Blood & Neg \\
\hline & 135 & Pos & Pos & 50 & 25 & 10 & 57 & 76 & & \\
\hline & 137 & Pos & Pos & 50 & 25 & 10 & 47 & 78 & & \\
\hline & 143 & Pos & Pos & 50 & 25 & 10 & 47 & 73 & & \\
\hline & 153 & Pos & Pos & 50 & 25 & 10 & 42 & 73 & & \\
\hline
\end{tabular}

a: months after admission, CFT: complement fixation test, reciprocal of titer, BPAT: Buffered plate agglutination, 2MET: 2-mercapto-ethanol, reciprocal of titer, RBT: Rose Bengal, SAT: Tube agglutination test, reciprocal of titer, CELISA cut-off \% $\% 28$, FPA: Fluorescence polarization assay cut-off $\mathrm{mP}>72$, Neg: Negative, Pos: Positive, Pos+/-: weakly positive, E: Error

B. abortus biovar 1 was isolated from his blood 39 months after admission and at the end of the second treatment scheme the blood culture was negative. He complained of having had myalgias and fatigue 60 months after the first consultation and B. abortus biovar 1 was isolated from his blood while serologic tests indicated high titres. CELISA and FPA showed persistently high titres of $\operatorname{IgG}$ antibodies in these patients, indicating chronic disease or relapse.

Patients who relapsed: Case 11 (Table 3), a dairy farmer, presented symptoms such as arthralgia and hepato-splenomegaly at the moment of the consult and malaise and vomiting seven months after treatment; $B$. suis biovar 1 was again isolated in the blood culture. He was treated with a second course of antibiotic regimens. By 10 and 22 months after admission the blood cultures were negative and the titres of conventional serology declined, but antibodies detected by CELISA and FPA persisted. Case 12, a pig slaughterhouse worker with fever and arthralgias was prescribed a prolonged treatment because $B$. suis biovar 1 was isolated from blood at admission and from bone-marrow, blood and bone-marrow, three, five and seven months later. Twenty six months after admission, conventional serology showed low titres but primary binding assays remained positive. Case 13, a kill area abattoir worker, presented fever and myalgia at admission and $B$. abortus biovar 1 was isolated from his blood. Seven months later he suffered arthralgia and malaise and the strain was again found in his blood. Twenty four months later, CELISA and FPA indicated persistence of antibodies and because the symptoms also persisted a bone marrow culture was done, though the results were negative. Case 14, a van driver that transported animal carcasses with fever, malaise and myalgia, presented a second isolation of B. abortus biovar 1 from the blood culture after the end of his treatment. During the following twenty five months, CELISA and FPA titres declined slowly. Case 15 regularly attended to the pigs housed near her home and presented fever and weight loss as main symptoms; brucellosis was diagnosed by isolation of strain from the blood culture. Four months later, despite completion of the treatment, new systemic 
Am. J. Infect. Dis., 3 (1): 27-35, 2007

Table 2: $\quad$ Serologic tests in five cases of "chronic" brucellosis ${ }^{\mathrm{b}}$

\begin{tabular}{|c|c|c|c|c|c|c|c|c|}
\hline Case & $\begin{array}{l}\text { Date } \\
\text { Da }\end{array}$ & SAT & 2MET & CFT & CELISA & FPA & $\begin{array}{l}\text { Source } \\
\text { isolation }\end{array}$ & Strain \\
\hline \multirow[t]{8}{*}{6} & 0 & 400 & 200 & 320 & 71 & $\mathrm{E}$ & $\begin{array}{l}\text { Blood } \\
\text { Bone biopsia } \\
\text { Epidural abscess }\end{array}$ & $\begin{array}{l}\text { B.suis bio } 1 \\
\text { B.suis bio } 1 \\
\text { B.suis bio } 1\end{array}$ \\
\hline & 2 & 200 & 200 & 160 & 73 & E & Epidural abscess & Neg \\
\hline & 3 & & & & & & Blood & Neg \\
\hline & 4 & & & & & & Epidural abscess & Neg \\
\hline & 6 & 200 & 200 & 160 & 74 & 95 & Blood & Neg \\
\hline & 14 & 200 & 200 & 160 & 85 & 100 & & \\
\hline & 17 & 100 & 100 & 80 & 84 & 95 & & \\
\hline & 32 & 100 & 50 & 40 & 70 & 72 & & \\
\hline \multirow[t]{6}{*}{7} & 0 & 1600 & 800 & 640 & 71 & 176 & Blood & B. abortus bio 1 \\
\hline & 2 & 1600 & 800 & 1280 & 79 & 175 & Blood & Neg \\
\hline & 19 & 200 & 100 & 80 & 83 & 277 & & \\
\hline & 22 & 200 & 50 & 40 & 82 & 181 & Blood & Neg \\
\hline & 23 & 200 & 50 & 10 & 72 & 147 & & \\
\hline & 34 & 100 & 50 & 5 & 62 & 123 & & \\
\hline \multirow[t]{6}{*}{8} & 0 & 800 & 400 & 40 & 89 & 166 & Blood & Neg \\
\hline & 3 & 400 & 400 & 40 & 89 & 146 & & \\
\hline & 6 & 200 & 200 & 20 & 90 & 139 & & \\
\hline & 9 & 200 & 100 & 20 & 85 & 138 & Blood & B.abortus bio 1 \\
\hline & 13 & 200 & 100 & 20 & 87 & 135 & Blood & Neg \\
\hline & 20 & 200 & 50 & 20 & 83 & 125 & & \\
\hline \multirow[t]{7}{*}{9} & 0 & 800 & 800 & 320 & 81 & 198 & & \\
\hline & 1 & 800 & 800 & 640 & 87 & 208 & Blood & B. abortus bio 1 \\
\hline & 3 & 800 & 400 & 320 & 88 & 201 & Blood & Neg \\
\hline & 5 & 400 & 200 & 160 & 86 & 203 & Blood & Neg \\
\hline & 12 & 100 & 100 & 80 & 84 & 140 & Blood & Neg \\
\hline & 16 & 50 & 50 & 80 & 86 & 138 & Blood & $\mathrm{Neg}$ \\
\hline & 24 & 50 & 50 & 80 & 85 & 126 & Blood & Neg \\
\hline \multirow[t]{5}{*}{10} & 0 & 400 & 200 & 320 & 89 & 144 & & \\
\hline & 11 & 400 & 200 & 160 & 89 & 146 & Blood & Neg \\
\hline & 39 & 200 & 200 & 320 & 92 & 133 & Blood & B.abortus bio 1 \\
\hline & 56 & 200 & 100 & 160 & 92 & 145 & Blood & Neg \\
\hline & 60 & 200 & 100 & 160 & 91 & 138 & Blood & B.abortus bio 1 \\
\hline
\end{tabular}

See legend Table 1

${ }^{\mathrm{b}}$ : positive to RBT and BPAT

symptoms and bacteraemia was observed, finding the strain in blood. The strains were sent to our laboratory for confirmation and typing and a serum sample was requested. Forty months later, CELISA and FPA titres remained very high.

Patients having brucellosis presumptively caused by B. canis: Case 16 presented fever and hepatosplenomegaly as main symptoms after a laboratory accident and $B$. canis was recovered from the blood culture. Serology performed with whole cell and LPS of $B$. abortus was negative but screening and confirmatory tests using B. canis antigen were positive; following treatment the titres declined slowly. Case 17 was a boy admitted to the hospital with fever and enlargement of liver and spleen, from whose blood B. canis was recovered, probably infected by contact with diseased dogs. The response to treatment was effective and 8 months later he had serologically and clinically recovered. Case 18, a boy admitted to the hospital with fever and hepato-splenomegaly, was diagnosed by serology because the blood culture was negative, probably because he was treated rapidly with antibiotics when his dogs had canine brucellosis. Cases 19 and 20 belonged to a family that was studied serologically, bacteriologically and clinically, because they were in contact with a dog with a history of canine brucellosis. They were asymptomatic at the time of the consultation, but the increase in the titres of antibodies was an alert and they were called in for repeated studies.

Patients with brucellosis that evolve during long period: Cases 21, 22 and 23 (Table 5) were two men 51 and 69 years old and a woman 69, admitted to different hospitals, whose titres to agglutination tests were low but had severe clinical symptoms. $B$. melitensis biovar 1 and $B$. suis biovar 1 were found in the blood cultures 
Am. J. Infect. Dis., 3 (1): 27-35, 2007

Table 3: Brucellosis serologic tests in five patients who relapsed

\begin{tabular}{|c|c|c|c|c|c|c|c|c|c|c|}
\hline Case & $\underset{\text { a }}{\text { Date }}$ & BPAT & RBT & SAT & 2MET & CFT & CELISA & FPA & $\begin{array}{l}\text { Source } \\
\text { isolation }\end{array}$ & train \\
\hline \multirow[t]{5}{*}{11} & 0 & Pos & Pos & 3200 & 400 & 640 & 87 & 189 & Blood & B. suis bio 1 \\
\hline & 3 & Pos & Pos & 800 & 50 & 160 & 72 & 115 & Blood & Neg \\
\hline & 7 & Pos & Pos & 400 & 50 & 40 & 84 & 234 & Blood & B.suis bio 1 \\
\hline & 10 & Pos & Pos & 100 & Neg & 5 & 80 & 156 & Blood & Neg \\
\hline & 22 & Neg & Pos+/- & 25 & Neg & Neg & 70 & E & Blood & Neg \\
\hline \multirow[t]{6}{*}{12} & 0 & Pos & Pos & 1600 & 400 & 320 & 77 & 173 & Blood & B.suis bio 1 \\
\hline & 3 & Pos & Pos & 400 & 400 & 40 & 79 & 161 & Bone-marrow & B.suis bio 1 \\
\hline & 5 & & & & & & & & Blood & B.suis bio 1 \\
\hline & 7 & & & & & & & & Bone-marrow & B.suis bio 1 \\
\hline & 18 & Pos & Pos & 50 & Neg & 5 & 34 & 87 & Blood & Neg \\
\hline & 26 & Neg & Neg & 50 & Neg & 5 & 46 & 93 & & \\
\hline \multirow[t]{7}{*}{13} & 0 & Pos & Pos & 12800 & 400 & 320 & 87 & $\mathrm{E}$ & Blood & B. abortus bio 1 \\
\hline & 7 & Pos & Pos & 800 & 200 & 160 & 84 & 154 & Blood & B. abortus bio 1 \\
\hline & 9 & Pos & Pos & 400 & 200 & 40 & 80 & 133 & Blood & Neg \\
\hline & 17 & Pos & Pos & 800 & 400 & 40 & 93 & 145 & & \\
\hline & 24 & Pos & Pos & 400 & 400 & 40 & 93 & 149 & & \\
\hline & 26 & & & & & & & & Bone-marrow & Neg \\
\hline & 29 & Pos & Pos & 400 & 200 & 40 & 90 & 137 & & \\
\hline \multirow[t]{6}{*}{14} & 0 & Pos & Pos & 6400 & 1600 & 320 & 96 & 174 & Blood & B. abortus bio 1 \\
\hline & 4 & Pos & Pos & 1600 & 800 & 320 & 70 & 163 & Blood & Neg \\
\hline & 5 & Pos & Pos & 3200 & 800 & 640 & 94 & 171 & Blood & B. abortus bio 1 \\
\hline & 9 & Pos & Pos & 1600 & 800 & 80 & 90 & 170 & Blood & Neg \\
\hline & 13 & Pos & Pos & 800 & 400 & 40 & 85 & 156 & & \\
\hline & 25 & Pos & Pos & 400 & 100 & 10 & 75 & 110 & & \\
\hline \multirow[t]{5}{*}{15} & 0 & & & & & & & & Blood & B.suis bio $1^{\mathrm{a}}$ \\
\hline & 4 & & & & & & & & Blood & B.suis bio $1^{\mathrm{a}}$ \\
\hline & 12 & Pos & Pos & 200 & 50 & 20 & 79 & 155 & & \\
\hline & 17 & Pos & Pos & 100 & 50 & 10 & 79 & 118 & & \\
\hline & 40 & Pos & Pos & 100 & Neg & 5 & 85 & 177 & & \\
\hline
\end{tabular}

See legend Table 1

Table 4: Serologic tests in five cases of brucellosis presumtively caused by $B$. canis $^{c}$

\begin{tabular}{|c|c|c|c|c|c|c|c|}
\hline Case & $\begin{array}{l}\text { Date } \\
\text { a }\end{array}$ & CELISA & FPA & RSAT & IELISA & $\begin{array}{l}\text { Source } \\
\text { isolation }\end{array}$ & Strain \\
\hline \multirow[t]{3}{*}{16} & 0 & 16 & 45 & 16 & 63 & Blood & B. canis \\
\hline & 3 & 23 & 48 & 8 & 41 & & \\
\hline & 5 & 19 & 52 & 2 & 33 & & \\
\hline \multirow[t]{3}{*}{17} & 0 & 11 & 40 & 32 & 87 & Blood & B. canis \\
\hline & 2 & 10 & 53 & 16 & 73 & & \\
\hline & 8 & 17 & 58 & 2 & 36 & & \\
\hline \multirow[t]{4}{*}{18} & 0 & 27 & 45 & 4 & 100 & Blood & Neg \\
\hline & 1 & 19 & 45 & 4 & 100 & & \\
\hline & 3 & 18 & 43 & 2 & 100 & Blood & Neg \\
\hline & 8 & 10 & 40 & Pos+/- & 75 & & \\
\hline \multirow[t]{3}{*}{19} & 0 & 12 & 62 & Pos+/- & 43 & Blood & Neg \\
\hline & 1 & 12 & 59 & 2 & 46 & & \\
\hline & 2 & 14 & 61 & 4 & 48 & & \\
\hline \multirow[t]{2}{*}{20} & 0 & 19 & 60 & Neg & 27 & Blood & Neg \\
\hline & 2 & 18 & 62 & 4 & 29 & & \\
\hline
\end{tabular}

${ }^{\mathrm{c}}$ : negative to BPAT, RBT, SAT, 2MET and CFT

See legend Table 1

RSAT: Rapid slide agglutination test

IELISA cut-off $\% \mathrm{P}>27$

and bone biopsy of cases 21 and 22, respectively, while the blood culture of case 23 was negative. Case 21 had lived on a farm all her life, where the consumption of unpasteurized dairy products was common; cases 22 and 23 had worked in abattoirs for many years (50 and 15 years). 
Am. J. Infect. Dis., 3 (1): 27-35, 2007

Table 5: Serologic tests in three patients with brucellosis that evolved during long period

\begin{tabular}{lllllllllll}
\hline Case & Date & BPAT & RBT & SAT & 2MET & CFT & CELISA & FPA & $\begin{array}{l}\text { Source } \\
\text { isolation }\end{array}$ & Strain \\
\hline 21 & 0 & Pos & Pos & 25 & Neg & 20 & 71 & E & Blood & B. melitensis bio 1 \\
22 & 0 & Pos $+/-$ & Neg & 25 & Neg & 40 & 88 & 155 & Bone biopsy & B.suis bio 1 \\
23 & 0 & Neg & Neg & Neg & Neg & 10 & 37 & 88 & Blood & Neg \\
\hline
\end{tabular}

\section{DISCUSSION}

Human brucellosis is a systemic infection characterized by variations in clinical signs and a multitude of somatic complaints. Complications with involvement of a single organ occasionally occur, in which case the disease is termed localized ${ }^{[13]}$. Serologic and bacteriologic tests are the tools for confirming the disease when it is suspected. The infection can be caused by B. suis, B. abortus and B. melitensis, although $B$. canis could also be implicated. Though diagnosis is accurate when the causal agent is isolated, this procedure is not always successful. Serologic tests have been developed as indirect proof of infection and to monitor the clinical cure. Some authors ${ }^{[1]}$ suggest that complete recovery from infection is normally followed up by a sharp reduction in antibody levels. A break in the reduction, replaced by a new increment in IgG levels is highly suggestive of a relapse or incomplete recovery.

The estimated prevalence of bovine brucellosis in Argentina is $10-13 \%$ of farm animals, with an individual rate of $4-5 \%$, the disease having been found in porcine, caprine, ovine and canine species ${ }^{[14]}$. At the NHBN we receive a large number of patients for diagnosis of brucellosis from whom $B$. abortus, $B$. suis, B. melitensis and B. canis have been isolated ${ }^{[15]}$. We have selected five groups of this patients that represent only a fraction of cases studies over the last 13 years. All sera were routinely tested with standardized tests previously reported ${ }^{[4-7,10]}$ and the primary objective of our study was to assess which diagnostic test was most effective in detecting different stages of the disease.

Brucellosis has been recognized as one of the most common laboratory-transmitted infections and has been reported to occur in clinical, research and production laboratories ${ }^{[16-19]}$. Usually, exposures result from unsafe laboratory practices such us sniffing plates, working on an open bench or not using protective clothes, mainly because aerosolization is the mechanism of transmission in this setting ${ }^{[20-22]}$. Though there seems to be growing concern about safe laboratory practices in Argentina, personnel of all microbiology laboratories should be trained in the proper use of equipment and procedures, including the recording of accidents ${ }^{[23]}$.
Table 1 shows five cases of laboratory-acquired brucellosis that we diagnosed, two were caused by $B$. melitensis biovar 1 , two by $B$. suis biovar 1 and one by $B$. suis biovar 1a. The third strain was resistant to dyes in a manner atypical of this species and is frequently isolated in the country ${ }^{[15]}$. Cases 1 to 3 have presented good evolution and an effective response to therapy and serologic variations were detected by both conventional and modern tests; however, the seroconversion of case 4 decreased more slowly. Serologic changes detected by classical tests in case 5 provided no information about the stage of infection, whereas CELISA and FPA with persistent high titres indicated infection activity. The case was given another treatment scheme one year after onset of the symptoms. We agree with some authors who have reported that most cases of laboratory-acquired brucellosis have shown the entire range of clinical manifestations of the disease ranging from the common prolonged febrile syndrome to focal signs and symptoms ${ }^{[21,24]}$.

No uniform definition has been agreed upon for the term "chronic" brucellosis and some authors have considered this classical categorization to be of limited clinical interest ${ }^{[25]}$; it is applied to patients with a history of symptoms extending over several months or complaints of ill health for more than one year after the diagnosis ${ }^{[26]}$. We present five cases followed up for a long period, one of them caused by B. suis biovar 1 and the other four cases by B. abortus biovar 1 (Table 2). Lumbalgias, arthralgias and myalgias were observed in the most of this patients. Osteoarticular complications are common in brucellosis having been reported to occur in $10-85 \%$ of cases ${ }^{[27-30]}$. Persistence of CELISA and FPA titres were observed in all the cases.

Relapse usually occurs in the first year after infection but varies widely according to length of treatment and drugs used ${ }^{[31]}$. Failure to complete therapy appears to be the principal cause of relapse, together with localized foci of infection that require surgical drainage ${ }^{[13]}$. As cause of relapse initial infection has been suggested to present certain characteristics depending on sex, bacteraemia and thrombocytopenia ${ }^{[32]}$. With few exceptions relapse is not due to the emergence of antibiotic resistant strains of Brucella ${ }^{[33]}$. From the five patients selected, three 
had positive isolation 7 months after admission and two relapsed at $4^{\text {th }}$ and $5^{\text {th }}$ month.

As with human infection by other species of Brucella, B. canis infections range from sub-clinical to the bacteriemic form. Diagnosis is generally made by isolating the strain from blood culture; and in most cases the source of infection is thought to be contact with infected dogs ${ }^{[26]}$. Since NHBN is engaged in diagnosing patients with symptoms and or epidemiology compatible with the disease, we study cases with negative serologic tests to smooth-Brucella abortus antigen because they could potentially be infected with $B$. canis. Table 4 shown five cases of brucellosis presumptively caused by $B$. canis, not detected by the classical serologic tests.

Also, this classical serologic tests may be not appropriate for detecting forms of the disease that evolve over long periods of time ${ }^{[34]}$ as are shown in Table 5. Negative blood cultures in the "chronic" form of the disease have been reported to be frequent ${ }^{[34]}$.

We selected these 23 cases in order to discuss the contribution of serologic methods to patient management. The BPAT is a practical screening test that reduces non specific reactions and is a bit more sensitive than RBT. SAT detects the acute form of the disease but cross reacts with other antibodies, its diagnostic specificity is poor and the diagnostic endpoint titre has not been satisfactorily established. $2 \mathrm{MET}$ is a reducing agent that, when added to the serum sample, reduces the IgM disulfide bridges into monomeric units, increasing the test's specificity but also possibly causing false negative reactions because IgG contains disulfide bridges that may be reduced. Because of its toxicity and the scant information it can add to the diagnosis of human brucellosis, its use should be discontinued.

The classical agglutination tests valuable for the acute form of the disease seem to be unsuitable for cases of relapse and the "chronic" form, which occur various months after the treatment. In the latter cases, the antibody levels detected by agglutination tests decrease over time and do not reflect the clinical symptoms.

CFT mainly identifies IgG antibodies that appear in the later stage of the disease or in the "chronic" form, but this test has several important disadvantages: it is unable to detect the acute form, is technically complicated to run, it presents anti-complementary activity and requires very labile reagents.

Of the newer serologic tests, primary binding assays were developed to improve sensitivity and specificity. CELISA is simple to perform, has fewer cross reactions with antibodies to other microorganisms, its titres correlate well with the clinical course and it is useful to detect acute cases as well as persistence of infection due to relapse or the "chronic" form. FPA has been shown to be accurate for detecting antibodies to Brucella sp. Although this test is sensitive (96.1\%), specific (97.9\%) and easy to run, it did not work with some sera, marked as " $E$ " in Table 13 and 5, with high lipid content that caused light scatter. RSAT could be a suitable test for the diagnosis of B.canis human brucellosis and IELISA performed in all RSAT positive samples that were negative by $B$. abortus antigen could ensure diagnostic specificity and confirm the diagnosis.

\section{CONCLUSION}

Because of the frequency of unusual clinical presentation of the disease, the negative blood culture that have been reported to be common in infected patients and the low titres of agglutination reactivity in cases that evolve during long periods we suggest the use of BPAT for screening and CELISA as confirmatory tests for diagnosis and for proper monitoring the course of treatment of brucellosis caused by S-Brucella sp. and RSAT and IELISA for diagnosis of $B$. canis brucellosis. This protocol that uses robust tests would be of value to the surveillance and control the disease.

\section{ACKNOWLEDGEMENTS}

We are very grateful to Deborah B. Hasan for her assistance and to Dr. Klaus Nielsen from the Canadian Food Inspection Agency, Animal Research Institute, Ontario, Canada, for reviewing the manuscript.

\section{REFERENCES}

1. Gazapo, E., J. Gonzalez Lahoz, J.L. Subiza, M. Baquero, J. Gil and E.G. de la Concha, 1989. Changes in IgM and IgG antibody concentrations in brucellosis over time: Importance for diagnosis and follow up. J. Infect. Dis., 159: 219-25.

2. Diaz, R. and I. Moriyon, 1989. Laboratory techniques in the diagnosis of human brucellosis. In: Brucellosis: Clinical and Laboratory Aspect (Eds. E.J. Young and M.J. Corbel), pp: 73-84. CRC, Boca Raton, Fl.

3. Nielsen, K., 2002. Diagnosis of brucellosis by serology. Vet. Microbiol., 90: 447-459. 
4. Lucero, N.E., L. Foglia, S.M. Ayala, D. Gall and K. Nielsen, 1999. Competitive enzyme immunoassay for diagnosis of human brucellosis. J. Cl. Microbiol., 37: 3245-48.

5. Lucero, N.E., G.E. Escobar, S.M. Ayala, P. Silva Paulo and K. Nielsen, 2003. Fluorescence polarization assay for diagnosis of human brucellosis. J. Med. Microbiol., 52: 883-87.

6. Lucero, N.E., G.I. Escobar, S.M. Ayala and N.R. Jacob, 2005. Diagnosis of human brucellosis caused by Brucella canis. J. Med. Microbiol., 54: 457-461.

7. Lucero, N.E., G.I. Escobar, S.M. Ayala and G. Lopez, 2002. Sensitivity and specificity of an indirect enzyme-linked immunoassay for the diagnosis of Brucella canis infection in dogs. J. Med. Microbiol., 51: 656-660.

8. Centro Panamericano de Zoonosis/PAHO/WHO, 1981. Brucelosis: prueba de fijación de complemento. Nota Técnica no. 24. CEPANZO, Buenos Aires, Argentina.

9. Alton, G.G., L.M. Jones, R.D. Angus and J.M. Verger, 1988. Bacteriological and serological methods. In: Techniques for the brucellosis Laboratory (eds Institut National de la Recherche Agronomique), pp: 3-136. INRA, Paris.

10. Lucero, N.E. and E. Bolpe, 1998. Buffered plate antigen test as a screening test for the diagnosis of human brucellosis. J. Clin. Microbiol., 36: 1425-27.

11. Carmichael, L.L. and J.C. Joubert, 1987. A rapid slide agglutination test for the serodiagnosis of Brucella canis infection that employs a variant(M-) organism as antigen. Cornell Vet., 77: 3-12.

12. Corbel, M.J. and W.J. Brinley Morgan, 1984. Genus Brucella, Meyer and Shaw 1920, 173 AL, In: Bergey's Manual of Systematic Bacteriology, (Eds. N.R. Krieg and J.G. Holt), 1: 377-388. The Williams \& Wilkins Co., Baltimore, Md.

13. Young, E.J., 1995. An overview of human brucellosis. Clin. Infect. Dis., 21: 283-290.

14. Samartino, L., 2002. Brucellosis in Argentina. Vet. Microbiol., 90: 71-80.

15. Lucero, N.E., S.M. Ayala, G.I. Escobar and N.R. Jacob, 2007. Brucella isolated in humans and animals in Latin America from 1968 to 2006. Epidemiol. Infect. In press.

16. Arlett, P.R., 1996. A case of laboratory acquired brucellosis. B.M.J., 313: 1130-1132.

17. Martin-Mazuelos, E., M.C. Nogales, C. Florez, J.M. Gomez Mateos, F. Lozano and A. Sánchez, 1994. Outbreak of Brucella melitensis among microbiology laboratory workers. J. Clin. Microbiol., 32: 20352036.

18. Zervos, M.J. and G. Bostic, 1997. Exposure to Brucella in the laboratory. Lancet, 349: 651.

19. Robichaud, S., M. Libman, M. Behr and E. Rubin, 2004. Prevention of laboratory-acquired brucellosis. Clin. Infect. Dis., 38: 119-122.
20. Grammont-Cupillard, M., L. Berthet-Badetti and P. Dellamonica, 1996. Brucellosis from sniffing bacteriological cultures. Lancet, 348: 1733-1734.

21. Noviello, S., R. Gallo, M. Kelly, R.J. Limberger, K. De Angelis, L. Cain, B. Wallace and N. Dumas, 2004. Laboratory-acquired brucellosis. Emerg. Infect. Dis., 10: $1848-1850$.

22. Al-Aska, A.K. and A.H. Chagla, 1989. Laboratoryacquired brucellosis. J. Hosp. Infect., 14: 69-71.

23. Lucero, N.E. and F. Siñeriz, 2005. The Argentine experience in enhancing biosafety through good laboratory practices. Asian Biotech. and Develop. Rev., 8: 99-120.

24. Yagupsky, P. and J.O. Baron Ellen, 2005. Laboratory exposure to Brucellae and implications for bioterrorism. Emerg. Infect. Dis., 11: 1180-1185.

25. Pappas, G., N. Akritidis, M. Bosilkovsky and E. Tsianos, 2005. Brucellosis. N. Engl. J. Med., 352: $2325-2336$

26. Young, E.J., 1983. Human brucellosis. Rev. Infect. Dis., 5: 821-842.

27. Gotuzzo, E., G.S. Alarcón, T.S. Bocanegra, C. Carrillo, J.C. Guerra, I. Rolando and L.R. Espinoza, 1982. Articular involvement in human brucellosis: a retrospective analysis of 304 cases. Semin. Arthritis Rheum., 12: 245-255.

28. Mousa, A.R., S.A. Muhtaseb, D.S. Almudallal, S.M. Khodeir and A.S. Marafie, 1987. Osteoarticular complications of brucellosis: a study of 169 cases. Rev. Infect. Dis., 9: 531-543.

29. Zaks, N., S. Sukenik, M. Alkan, D. Flusser, L. Neumann and D. Buskila, 1995. Musculoskeletal manifestations of brucellosis: a study of 90 cases in Israel. Semin. Arthritis Rheum., 25: 97-102.

30. Gonzalez-Gay, M.A., C. Garcia-Porrua, D. Ibáñez and M.J. Garcia-Paiz, 1999. Osteoarticular complications of brucellosis in an Atlantic area of Spain. J. Rheumatol., 26: 141-45.

31. Solera, J., E. Martinez-Alfaro, A. Espinoza, M.L. Castillejos, P. Guijo and M. Rodríguez-Zapata, 1998. Multivariate model for predicting relapse in human brucellosis. J. Infect., 36: 85-92.

32. Ariza, J., J. Corredoira, R. Pallares, P.F. Viladrich, G. Rufi, M. Pujol and F. Gudiol, 1995. Characteristics of and risk factors for relapse of brucellosis in humans. Clin. Infect. Dis., 20: 1241-1249.

33. Ariza, J., J. Bosch, F. Gudiol, J. Liñares, P. Fernandez-Viladrich and R. Martin, 1986. Relevance of in vitro antimicrobial susceptibility of Brucella melitensis to relapse rate in human brucellosis. Antimicrob. Agents Chemother., 30: 958-960.

34. Ariza, J., C. Pigrau, C. Cañas, A. Marron, F. Martinez, B. lmirante, J.M. Corredoira, A. Casanova, J. Fabregat and A. Pahissa, 2001. Current understanding and management of chronic hepatosplenic suppurative brucellosis. Clin. Infect. Dis., 32: 1024-1033. 